\title{
Notes on implementing a IEEE 802.11s Mesh Point
}

\author{
Rosario G. Garroppo, Stefano Giordano, Davide Iacono, Luca Tavanti \\ Dip. Ingegneria dell'Informazione \\ Università di Pisa, Italy \\ \{name.surname\}@iet.unipi.it
}

\begin{abstract}
Keywords - Wireless Mesh Networks, IEEE 802.11s, HWMP, prototype, experimental testbed.
\end{abstract}

\section{EXTENDED ABSTRACT}

Wireless Mesh Networks (WMNs) are gaining wide popularity as a flexible and cost-effective alternative to the traditional set of disjointed IEEE 802.11 Access Points (APs). Their all-wireless infrastructure is in fact suitable for both residential premises and for hardly accessible places. As a consequence, many companies have already put on the market their own WMN solutions. Yet, though most of them are based on the common 802.11 MAC [1], these products are not interoperable. To harmonize this technology, IEEE set up the 802.11s working group (TGs) whose goal is defining a standard architecture for WMNs. Unfortunately, the drafting of the standard has turned out to be rather troublesome, many issues are still open, and proposals are being submitted to TGs in order to complete and improve the upcoming standard. So far the proposed solutions have been evaluated analytically or through simulations, but there is no experimental evidence of their goodness.

These reasons motivated us to start an implementation of a IEEE 802.11 s compliant node to experimentally verify the effectiveness of the current draft standard and its possible enhancements. We mostly dealt with the path selection and frame forwarding facilities, which are two of the main innovations of 802.11s. Implementing them were very challenging tasks. We had to face several issues that drove us to develop solutions not strictly adhering to the draft. Some issues were related to the available hardware and software tools (for example, modifying the basic 802.11 frames and creating the six-address Mesh Data frames was not feasible), while others came directly from the 802.11s draft in the form of missing, vague, or even faulty indications (mostly about frame forwarding and station management).

In the paper, instead of giving all details of our prototype, we prefer highlighting the implementation issues we had to face and how we solved them. For the same reason, we limit the overview of 802.11s (and the proposals in [2]) just to the aspects related to our work. More details about the unofficial draft can be found in [3].

\section{A. Implemented architecture and features}

The basic 802.11 s network entity is the Mesh Point (MP). Beyond having all the characteristics of a traditional 802.11 station, every MP is also called to relay the traffic generated by other MPs to enable them to reach the intended destination through a multi-hop path. The set of connections among the MPs forms the wireless backbone of the Mesh. A MP can also have additional features, such as gateway/bridging functions, to connect to an external network, like the Internet. In this case, the MP is called Mesh Point Portal (MPP), or just Portal. A 802.11s network may have one or more Portals, and each MP chooses which Portal to use to get access to the external world. Another option for a MP is to give legacy client stations (STAs) access to the Mesh. In this case a MP becomes a Mesh Access Point (MAP) and must offer all the functions provided by the basic 802.11 standard. From the STA point of view, the Mesh must be completely transparent.

We implemented a prototype 802.11s Mesh Point using common hardware (laptop and desktop PCs) and open source software (Linux). We exploited the Multiband Atheros Driver for Wi-Fi (MadWifi) [6], which provides for the creation of several Virtual Access Points (VAPs). All VAPs work with the same physical device, but each VAP appears to the operating system as different wireless interface. Then, the Linux bridge module was used to connect the interfaces created by MadWifi. This module behaves exactly like a hardware bridge, forwarding on its ports the frames according to their destination MAC address. The resulting framework is shown in Figure 1.

The system appears as an 802.11s software overlaid above the legacy 802.11 hardware and software. Several VAPs have been set up in different modes in every MP. The ap VAP supports the interaction with user stations as defined in the basic 802.11 standard. The $w d s$ VAPs are used to form the WMN backbone. Each $w d s$ VAP builds a single static link to another MP using all the four addresses of the 802.11 frame header with the "from DS" and "to DS" flags set. Every ap and $w d s$ VAP is connected to a port of the bridge, that forwards the frames between the interfaces. Finally, the two monitor VAPs are used to send and receive the new 802.11s management frames. With this solution we exploit the legacy 802.11 Wireless Distribution System adding it the new Mesh services. Hence our framework agrees with the draft indications of having a transparent Mesh layer.

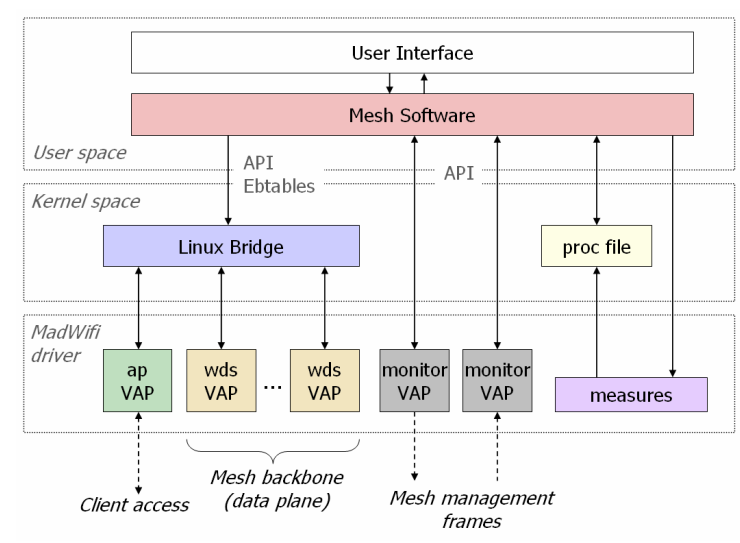

Figure 1. Architecture of the implemented software framework.

To build the set of paths that form the Mesh backbone, 802.11 s brings routing at level two of the OSI stack. This technique makes the Mesh transparent to upper layer protocols (e.g. IP), that see any intended destination within 
the Mesh to be only one hop away. The Hybrid Wireless Mesh Protocol (HWMP) is the mandatory algorithm that all MPs must implement to guarantee the full functioning of the Mesh. HWMP combines an on-demand algorithm with a proactively built tree topology. The second technique is used when at least one Portal is present in the network, since the tree shall be rooted at the Portal. Both the on-demand and the proactive techniques use common messages and processing rules. The Route Request (RREQ), Route Reply (RREP), Route Error (RERR) and Root Announcement (RANN) frames are flexibly structured to allow for the needs of both protocols. These messages are transported in the new Mesh Management frames.

The HWMP tree connects all MPs to the Portal (root), so that a path is always available towards the outside and between all MPs. It can be set up in two ways. The root can broadcast either Proactive RREQ (PRREQ) or RANN frames. The first technique aims at creating and maintaining a set of paths towards the root from all MPs. A MP receiving the PRREQ replies with a unicast Gratuitous RREP (GRREP), where it also inserts the address of its children MPs that use it to reach the root (dependent nodes). In this way the Portal is able to re-construct the full topology of the network. The second technique just disseminates information on how the root can be reached, leaving each MP the possibility to set up the path whenever it needs it (basically using the on-demand algorithm). Both PRREQ and RANN are re-broadcasted by each MP.

The HWMP on-demand algorithm works much like AODV [4]. A source Mesh Point S wanting to send data to a destination MP D broadcasts a RREQ frame indicating the MAC address of D. Two flags specify the handling policy of the frame: DO (Destination Only) and RF (Reply and Forward). If the DO flag is set, only the destination is allowed to reply to the RREQ, otherwise any intermediate node having a path to D can answer to S's request. If RF is set, intermediate nodes may reply to $S$, but shall nevertheless re-broadcast the RREQ. In any case, all nodes receiving the RREQ can add or update their path to S. Once D (or any allowed intermediate node) receives the RREQ, it sends $S$ a unicast RREP. Intermediate nodes shall then forward this RREP to $S$ along the best path, and, when the RREP reaches $\mathrm{S}$, the path is set up and can be used for exchanging data.

We designed our prototype so that a HWMP tree is built through the PRREQ/GRREP messages and used as the main transport infrastructure, whereas on-demand paths are set up for connections among stations within the mesh. We also optimized the on-demand procedure so that RREQ flooding is reduced. In particular, each node, instead of rebroadcasting all the received RREQs (coming from different paths), it merges them into a unique RREQ that is eventually sent out. This considerably reduces the protocol overhead.

\section{B. Implementing issues}

One of the main hurdles to overcome was the scarce weight that $802.11 \mathrm{~s}$ gives to the fact that stations are the main sources and sinks of data frames. The format of the Mesh Management frame was in fact designed for communications between MPs. As a result, managing stations with frames not designed for this purpose was a very problematic situation. That is why we used the frame format as defined in [3], which adds an extra address field to account for any station the MAP acts on behalf of (in particular, the new RREQ frame). For the same reason we also integrated in the framework the Proxy mechanisms proposed in [3]. In short, each MAP shall act as a Proxy for its associated STAs. It handles and converts the frames that enters or exit the Mesh, and it casts Proxy Update (PU) messages to inform the other MPs (actually, only the MPP) about its associated stations.

Still according to the original 802.11s draft, all MPs shall store the address of every station in the network with the corresponding MAP. This behaviour is the key to perform many procedures involving a station (e.g. an on-demand path discovery). However, this method is rather cumbersome, so we found a simpler way in putting all the information in the Portal using the PU messages sent by the MAPs. This is coherent with the assumption that, since the final users of the Mesh (as an access technology) are principally consumers, most traffic is addressed to or comes from the Portal, which represents the gateway to the Internet. The root is then a privileged point for collecting and distributing information on the network.

Finally, to select the best paths, the $802.11 \mathrm{~s}$ draft defines the mandatory "airtime" metric (though other metrics can be used). The airtime metric is very similar to the well known ETX metric [5]. The definition is the following:

$$
C_{a}=\left[O+\frac{B_{t}}{r}\right] \frac{1}{1-e_{p t}}
$$

In the formula, $O$ is a constant that quantifies the channel access and protocol overhead, $B_{t}$ is the test frame length (in bits), $r$ is the transmission rate (in Mbps), and $e_{p t}$ is the test frame loss ratio. MPs should continuously monitor and probe their links to keep the metric up to date with the current network state.

The airtime metric turned out to be very sensitive to changes in link usage. We noticed the occurrence of a perilous "ping-pong" effect among paths with similar metrics. It may happen that the unloaded (or just less loaded) path momentarily offers a better metric, so a MP is induced to select that path to convey the traffic. Shortly, however, the new route (now more loaded) will suffer a metric degradation at the expenses of the old path, which, on the contrary, is now unloaded. The MP is therefore bound to change again, thus creating an oscillating phenomenon. Since each path change has some overhead, network resources may be significantly reduced. We addressed the problem with a slight change in the metric (the transmission rate was replaced by the signal to noise ratio) to lessen the dependence on the frame loss percentage. Then we also used a moving average, to smooth out the effects of sudden rises and falls in the metric, and forced the MPs to wait for a stable situation (i.e. a number of messages announcing a better metric) before switching to a new path.

\section{REFERENCES}

[1] IEEE Standard 802.11-2007, June 2007.

[2] IEEE P802.11s, "Proxy Frame Forwarding", IEEE document 802.1107/0337r1, March 2007.

[3] G. R. Hiertz, S. Max, R. Zhao, D. Denteneer, L. Berlemann, "Principles of IEEE 802.11s", Proc. of $16^{\text {th }}$ International Conference on Computer Communications and Networks (ICCCN), August 2007.

[4] C. Perkins, E. Belding-Royer, S. Das, "Ad hoc On-Demand Distance Vector (AODV) Routing”, IETF RFC 3561, July 2003.

[5] D. S. J. De Couto, D. Aguayo, J. Bicket, R. Morris, "A HighThroughput Path Metric for Multi-Hop Wireless Routing", ACM Mobicom, 2003.

[6] Multiband Atheros Driver for Wi-Fi, at: http://madwifi.org. 